\title{
Cadherin 17 is related to recurrence and poor prognosis of cytokeratin 19-positive hepatocellular carcinoma
}

\author{
CHAO-WEI LEE ${ }^{1-3}$, SEY-EN LIN ${ }^{4}$, HSIN-I TSAI ${ }^{2,3,5}$, PO-JUNG SU ${ }^{2,6,7}$, CHIA-HSUN HSIEH $^{2,6}$, \\ YUNG-CHIA KUO ${ }^{2,3,6}$, CHANG-MU SUNG $^{2,3,8}$, CHENG-YU LIN $^{8}$, CHI-NEU TSAI $^{3}$ and MING-CHIN YU ${ }^{1-3}$ \\ ${ }^{1}$ Department of Surgery, Chang Gung Memorial Hospital, Taoyuan 33305; ${ }^{2}$ College of Medicine; \\ ${ }^{3}$ Graduate Institute of Clinical Medical Sciences, Chang Gung University, Taoyuan 33302; ${ }^{4}$ Department of Pathology, \\ Wan Fang Hospital, Taipei Medical University, Taipei 11696; Departments of ${ }^{5}$ Anesthesiology and ${ }^{6}$ Hematology-Oncology, \\ Chang Gung Memorial Hospital, Taoyuan 33305; ${ }^{7}$ Graduate Institute of Data Science, Taipei Medical University, Taipei 11031, \\ ${ }^{8}$ Department of Gastroenterology and Hepatology, Chang Gung Memorial Hospital, Taoyuan 33305, Taiwan, R.O.C.
}

Received March 22, 2017; Accepted October 20, 2017

DOI: $10.3892 / \mathrm{ol} .2017 .7320$

\begin{abstract}
A previous study demonstrated that cytokeratin 19 (CK19) expression in hepatocellular carcinoma (HCC) is an indicator of HCC invasiveness, including lymph node metastasis (LNM), tumor infiltration/non-encapsulation and poor prognosis. The exact mechanism by which CK19 expression results in poor prognosis remains unclear. Through the use of an Affymetrix U133A oligonucleotide microarray [20 patients with hepatitis B virus (HBV)-HCC], it was demonstrated that cadherin 17 (CDH17) significantly correlated with CK19 expression $\left(\mathrm{R}^{2}, 0.867 ; \mathrm{P}<0.001\right)$ in HBV-HCC. Immunohistochemical analysis (114 patients with HBV-HCC) also demonstrated a significant correlation between CK19 and $\mathrm{CDH} 17$ expressions in primary tumor tissue $\left(\mathrm{R}^{2}, 0.414\right.$; $\mathrm{P}<0.001)$. In addition, CK19 and CDH17 expressions levels revealed a significant association with LNM $(\mathrm{P}<0.001)$. Cox regression multivariate analysis demonstrated that indocyanine green retention at $15 \mathrm{~min}>10 \%$ and $\mathrm{CDH} 17$ expression were independent prognostic factors for disease free survival $(\mathrm{P}=0.010$ and 0.002 , respectively). In vitro studies showed that epidermal growth factor can induce the expression of both
\end{abstract}

Correspondence to: Dr Ming-Chin Yu, Department of Surgery, Chang Gung Memorial Hospital, 5 Fuxing Street, Guishan, Taoyuan 33305, Taiwan, R.O.C.

E-mail: mingchin2000@gmail.com

Abbreviations: AFP, $\alpha$-fetoprotein; AJCC, American Joint Committee on Cancer; CDH17, cadherin 17; CEA, carcinoembryonic antigen; CK19, cytokeratin 19; CT, computed tomorgraphy; DFS, disease-free survival; EGF, epidermal growth factor; EGFR, epidermal growth factor receptor; HBV, hepatitis B virus; HCC, hepatocellular carcinoma; HCV, hepatitis C virus; ICG-15, indocyanine green retention at $15 \mathrm{~min}$; IHC, immunohistochemistry; LNM, lymph node metastasis; OS, overall survival; qRT-PCR, quantitative real-time PCR

Key words: cytokeratin 19, keratin 19, cadherin 17, hepatocellular carcinoma, hepatoma, recurrence, prognosis
CK19 and CDH17, and CDH17 in turn can enhance the expression of CK19 in HCC. In summary, this study demonstrated that the early recurrence and poor prognosis of CK19(+) HCC may be due to the expression of $\mathrm{CDH} 17$, a gene known to be associated with vascular invasion, tumor metastasis, and advanced tumor stage of HCC. Thus, novel therapeutics by targeting CDH17 may be beneficial for CK19(+) HCC.

\section{Introduction}

Hepatocellular carcinoma (HCC) is the most common primary malignancy of the liver with an estimated annual death incidence of approximately 700,000 worldwide (1,2). In Taiwan, it is the second most common cause of cancer death and causes more than 7,500 deaths each year (3). Surgical resection remains the most effective therapy in selected patients, but approximately $75 \%$ of patients with HCC have advanced unresectable diseases upon presentation. In order to improve long-term outcome of patients with HCC, numerous efforts were made to unravel the risk factors for and pathogenesis of poor prognosis for HCC.

Cytokeratin 19 (CK19) is a biliary epithelial cell marker and is generally expressed in intrahepatic cholangiocarcinoma (ICC) cells $(4,5)$. Studies have shown that the expression of CK19 in primary HCC is associated with poorer outcome (6). There have also been studies demonstrating that CK19 expression in primary $\mathrm{HCC}$ is a significant risk factor for developing lymph node metastasis (LNM) $(4,7,8)$. Compared with other malignancies such as lung cancer, esophageal cancer, renal cancer, gastric cancer, and intra-hepatic cholangiocarcinoma, the incidence of LNM in primary HCC is low, and the prognosis is poor when LNM occurs (9-11). HCC with CK19 expression, subsequently, may be a special subtype of $\mathrm{HCC}$ with distinct clinical behavior from HCC without CK19 expression (8). Due to its worse prognosis, it is therefore of clinical significance to elucidate the pathogenesis of CK19-positive [CK19(+)] HCC. In the meanwhile, cadherin-17 (CDH17) was shown to be associated with vascular invasion and tumor metastasis in HCC (12). Similar to $\alpha$-fetoprotein (AFP), CDH17 is present only in fetal liver and gastrointestinal tract during embryogenesis, and the 
gene becomes silenced in healthy adult liver and stomach tissues. It functions as a peptide transporter and a cell adhesion molecule to maintain tissue integrity in epithelia (13). To date, there is little report to dissect the mechanistic relationship between CK19 and CDH17 in CK19(+) HCC. As a result, the purpose of this study was to investigate whether $\mathrm{CDH} 17$ is responsible for the invasiveness and poor prognosis of CK19(+) HCC.

\section{Materials and methods}

Patients. For the years from 2007 to 2012, records of patients with histological proven primary HCC from the Cancer Registry of the Cancer Center, Chang Gung Memorial Hospital (Taoyuan, Taiwan), were retrospectively reviewed. Since hepatitis B virus (HBV) infection remains the most common etiology of HCC in Taiwan (11), we recruited HBV-related HCC (HBV-HCC) first for the current study. The inclusion criteria of current study was defined as resectable lesions without distant metastasis by image study, including sonography, computed tomography (CT), and angiography. Only patients who received curative hepatectomy as initial primary treatment for HCC were included. Patients who had unresectable disease, synchronous cancers, recurrent cancers, distant metastasis or previous history of other malignancy preoperatively were excluded from the study. Their clinico-pathological data were retrieved from the prospectively collected database. The following variables were included in the analyses: Age, sex, cigarette smoking, alcohol consumption, HBV infection, anti-hepatitis $\mathrm{C}$ virus antibody (anti-HCV) level, alkaline phosphotase level, bilirubin level, preoperative AFP and carcinoembryonic antigen (CEA) level, Child-Pugh classification, tumor size, tumor-LNM status, tumor encapsulation, histological grade, vascular invasion, tumor rupture, daughter nodules, resection margin, and long-term survival. The study endpoint was 30 June 2015, and tumor staging was based on the 7th edition of American Joint Committee on Cancer (AJCC) TNM staging system for HCC (14). This study was approved by the Institutional Review Boards (IRB 103-2225C) of Chang Gung Memorial Hospital.

Immunohistochemistry (IHC). Formalin-fixed and paraffin-embedded resection specimens were sectioned to $4 \mu \mathrm{m}$ in thickness and deparaffininzed, rehydrated, and processed for antigen retrieval. The slides were further incubated with appropriate dilutions of the following antibodies $(\mathrm{Ab})$ at room temperature for $1 \mathrm{~h}$ (CK19 Ab; Abcam, San Francisco, CA, USA; and CDH17 Ab; LifeSpan Biosciences, Seattle, WA, USA). After incubation, the slides were washed 3 times in phosphate-buffered saline (PBS), incubated with a horse reddish peroxidase conjugated antibody polymer (Zymed; Thermo Fisher Scientific, Inc., Waltham, MA, USA) at room temperature for $10 \mathrm{~min}$, and then developed by treatment with 3,3'-diaminobenzidine (Roche Diagnostics, Basel, Switzerland) at room temperature for $10 \mathrm{~min}$. An independent experienced pathologist without knowledge of patient characteristics and outcome was asked to determine the results of immunohistochemical staining under microscopy. A positive result was defined as $\geq 10 \%$ of tumor cells stained positive for CK19 or CDH17.
Western blot analysis. Total protein was extracted from each cell line, boiled at $95^{\circ} \mathrm{C}$ for $5 \mathrm{~min}$, and placed in $10 \%$ SDS-polyacrylamide gel for electrophoresis. The protein images from the gel were transferred to a nitrocellulose membrane and blocked with 5\% nonfat milk in PBST solution (PBS plus 0.15 Triton X-100). The membrane was washed again and incubated again with anti-mouse immunoglobulin $\mathrm{G}$ antibody conjugated with horseradish. The blots were probed with antibodies specific for CK19 (Abcam) or CD17 (Lifespan Biosciences) and appropriate secondary antibodies. Labeled bands were subsequently detected by enhanced chemiluminescence (ECL; Millipore, Bedford, MA, USA). For each sample, band intensities were normalized to glyceraldehyde-3-phosphate dehydrogenase (GAPDH).

Quantitative real-time PCR ( $q R T-P C R)$. The first-strand cDNA was synthesized from $5 \mu \mathrm{g}$ of total RNA, and $0.5 \mu \mathrm{l}$ of cDNA will be mixed with Taqman probe (Assay-on-Demand; Applied Biosystems, Foster City, CA, USA. Cat. no. for CK19, Hs00761767_s1; for CDH17, Hs00900408_m1), RNase-free water, and TaqMan Universal PCR Master Mix. Real-time PCR was performed according to the manufacturer's protocol. GAPDH was used as internal controls for gene expression. For normalized each sample, the relative gene expression was calculated using the differences in threshold cycles (Delta $\mathrm{Ct}$, $\Delta \mathrm{Ct})$, a calibrated $\Delta \mathrm{Ct}$ value $\left(\Delta \mathrm{Ct}=\mathrm{Ct}\right.$ internal control $^{-} \mathrm{Ct}$ sample $)$. For quantitation of gene expression levels between treated and non-treated samples, the comparative $\mathrm{C}_{\mathrm{t}}$ method (also known as the $2^{-\Delta \Delta \mathrm{Ct}}$ method), where $\Delta \Delta \mathrm{C}_{\mathrm{t}}=\Delta \mathrm{C}_{\mathrm{t} \text { treated }}-\Delta \Delta \mathrm{C}_{\mathrm{t} \text {, non-treated }}$, was used.

Statistical analysis. The statistical analysis was performed with IBM SPSS Statistics 21 (IBM Corp., Somers, NY, USA). Fisher's exact test and Pearson's $\chi^{2}$ test were used to analyze categorical data. Student's t-test was used to analyze quantitative variables. Overall survival (OS) was defined by the time elapsing from the date of diagnosis to either the date of death or the date of the last contact. Disease-free survival (DFS) was calculated from the date of surgery to the date of the first documented clinical disease recurrence. Cases with surgical mortality, defined as death within one month of surgery, were excluded from the survival analyses. Kaplan-Meier analysis was used to determine the OS and DFS. The log-rank test and Cox regression multivariate analysis were applied to determine prognostic significance of clinicopathological variables. Statistical significance was defined as $\mathrm{P}<0.05$.

\section{Results}

Patient demographics. A total of $114 \mathrm{HBV}-\mathrm{HCC}$ patients who underwent liver resection were recruited for the current study. The median follow-up time was 49.02 months (mean, 52.4 months). The mean age of diagnosis was 55.7 year-old and about $80 \%$ patients were male. As for preoperative liver function, 108 patients (97.3\%) were Child-Pugh A, while only 3 patients $(2.7 \%)$ were Child-Pugh $\mathrm{B}$. The indocyanine green retention at $15 \mathrm{~min}(\mathrm{ICG}-15) \leq 10 \%$ were found in 75 patients (66.4\%; mean ICG-15, 9.62\%). The mean preoperative AFP and CEA levels were 10,581.94 and $3.14 \mathrm{ng} / \mathrm{ml}$, respectively. As for pathological variables, the mean tumor size was $4.18 \mathrm{~cm}$ 
Table I. Demographic data of patients with HBV-related hepatocellular carcinoma undergoing hepatectomy $(n=114)$.

A, Categorical variables

\begin{tabular}{|c|c|}
\hline Variables $^{\mathrm{a}}$ & No. $(\%)$ \\
\hline Age, $\leq 65$ years & 90 (78.9) \\
\hline Sex (male) & $90(78.9)$ \\
\hline \multicolumn{2}{|l|}{ Comorbidity } \\
\hline Diabetes mellitus (yes) & $19(16.8)$ \\
\hline Hypertension (yes) & $22(19.3)$ \\
\hline ESRD $^{\text {b }}$ (yes) & $1(0.9)$ \\
\hline Smoking (yes) & $21(18.4)$ \\
\hline Alcohol (yes) & $16(14.0)$ \\
\hline Child-pugh classification & $108(97.3) /$ \\
\hline$(\mathrm{A} / \mathrm{B} / \mathrm{C})$ & $3(2.7) / 0(0)$ \\
\hline $\begin{array}{l}\text { Preoperative } \alpha \text {-fetoprotein } \\
(>200 \mathrm{ng} / \mathrm{ml})\end{array}$ & $54(47.4)$ \\
\hline ICG-15 ( $\leq 10 \%)$ & $75(66.4)$ \\
\hline Tumor size $(>5 \mathrm{~cm})$ & $23(20.2)$ \\
\hline Tumor encapsulation (yes) & $93(81.6)$ \\
\hline Tumor rupture (yes) & $6(5.3)$ \\
\hline Vascular invasion (yes) & $29(25.4)$ \\
\hline Daughter nodules (yes) & $22(19.3)$ \\
\hline Resection margin (negative) & $113(99.1)$ \\
\hline Edmonson and steiner grade & $9(8.0) / 59(52.2) /$ \\
\hline (I/II/III/IV) & $40(35.4) / 5(4.4)$ \\
\hline Liver cirrhosis (yes) & $58(50.9)$ \\
\hline Tumor necrosis (yes) & $56(49.1)$ \\
\hline AJCC T stage & $66(69.5) / 18(18.9) /$ \\
\hline (T1/T2/T3a/T3b/T4) & $8(8.4) / 1(1.1) / 2(2.1)$ \\
\hline AJCC N stage (N0) & $113(99.1)$ \\
\hline
\end{tabular}

$\mathrm{B}$, Continuous variables

\begin{tabular}{lc}
\hline Variables $^{\mathrm{a}}$ & Mean \pm SE \\
\hline Age (years) & $55.7 \pm 1.09$ \\
ICG-15 (\%) & $9.62 \pm 1.02$ \\
Preoperative $\alpha$-fetoprotein $(\mathrm{ng} / \mathrm{ml})$ & $10,581.94 \pm 5,851.93$ \\
Preoperative CEA $(\mathrm{ng} / \mathrm{ml})$ & $3.14 \pm 0.73$ \\
Tumor size $(\mathrm{cm})$ & $4.18 \pm 0.24$ \\
\hline
\end{tabular}

${ }^{a}$ Only patients with available data were analyzed. ${ }^{b}$ End-stage renal disease. CEA, carcinoembryonic antigen; HBV, hepatitis B virus; ICG-15, indocyanine green retention at $15 \mathrm{~min}$; ESRD, end-stage kidney or renal disease; AJCC, American Joint Committee on Cancer; SE, standard error of the mean.

and 93 tumors $(81.6 \%)$ were encapsulated. About one-fourth of tumors had either micro- or macro-vascular invasion and 22 tumors $(19.3 \%)$ had daughter nodules. Tumor rupture was found in only 6 cases $(5.3 \%)$. R0 resection was achieved in the majority of patients $(99.1 \%)$. About half of the patients had histology-proven liver cirrhosis and most patients $(69.5 \%)$ had

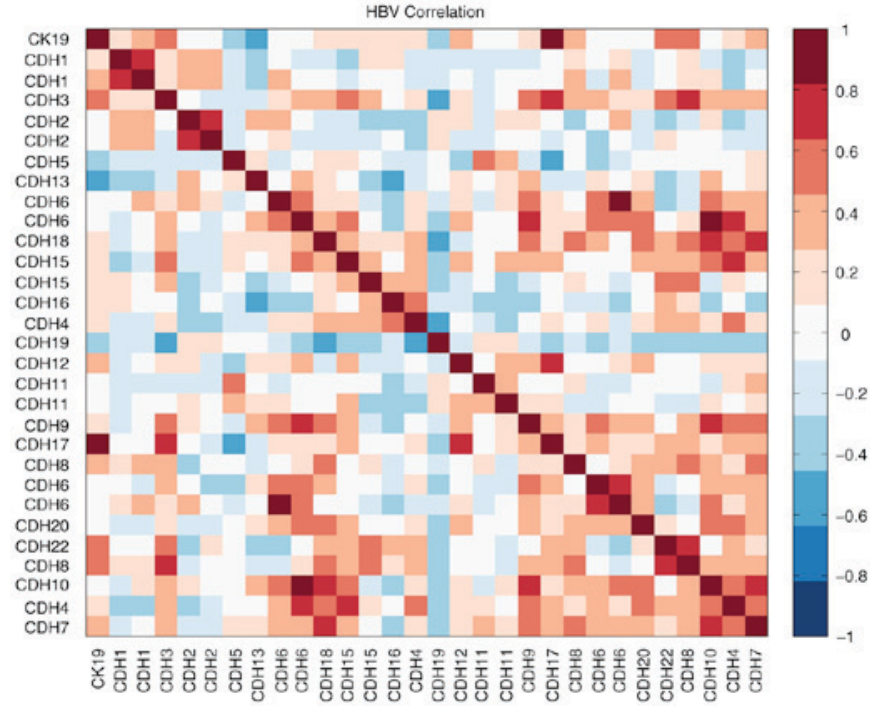

Figure 1. Heat map presentation of correlations between CK19 and various cadherins expressions after Affymetrix U133A oligonucleotide microarray analysis. Red colors represented positive correlation and blue colors represented negative correlation. The significance of correlation was determined by Pearson correlation coefficient $\mathrm{R}^{2}$. For $\mathrm{CDH} 17$, the $\mathrm{R}^{2}$ was $0.867, \mathrm{P}<0.001$. CK19, cytokeratin 19; CDH, cadherins; CDH17, cadherin 17; HBV, hepatitis B virus.

T1 disease. The clinico-pathological data were summarized in Table I.

Microarray data mining and immunohistochemical study. In our earlier study, we have established a dataset for early stage HBV-HCC $(<5 \mathrm{~cm})$ patients $(\mathrm{n}=20)$ by using Affymetrix U133A oligonucleotide microarray (15). We then took the advantage of this dataset and tried to search for potential genes that might be correlated with CK19 expression. After correlation analysis, we found at least one important molecule, CDH17, that was significantly correlated with CK19 expression in HBV-HCC $\left(\mathrm{R}^{2}: 0.867, \mathrm{P}<0.001\right.$; Fig. 1).

To further examine the correlation between CK19 and CDH17 expression, immunohistochemical (IHC) study was performed and the results were interpreted by experienced pathologists. CK19 expression was found in nine tumors (7.9\%), and five tumors (4.4\%) showed immunopositivity for CDH17. Three patients showed immuno-positivity for both CK19 and CDH17. While almost none of the CK19(-) HCC showed CDH17 expression (2 out of 104 patients, 1.9\%), one-third of CK19(+) HCC had simultaneous CDH17 expression. The Spearman's correlation coefficient was 0.414 and P-value was $<0.001$. As demonstrated in Fig. 2, the vast majority of the CK19(+) tumor cells in CK19(+) HCC also had strong CDH17 expression. The expression of CK19 seemed to coincide with that of CDH17 in these CK19(+) HCC.

Clinicopathological features and survival analysis of CK19(+) or CDH17(+) HCC. As shown in Table II, CK19 expression was significantly associated with LNM and CDH17 expression $(\mathrm{P}<0.001$, respectively). In addition, CK19(+) HCC was more likely to have elevated preoperative AFP > $200 \mathrm{ng} / \mathrm{ml}$. In the meanwhile, CDH17 expression was also significantly associated with LNM and CK19 expression 
Table II. The relationship between clinicopathological variables and CK19 expression in hepatocellular carcinoma $(n=114)$.

\begin{tabular}{|c|c|c|c|}
\hline & \multicolumn{3}{|c|}{ CK19a } \\
\hline & Negative $(\%)$ & Positive (\%) & P-value \\
\hline \multicolumn{4}{|c|}{ Age (years) } \\
\hline$\leq 65$ & $82(78.1)$ & $8(88.9)$ & 0.446 \\
\hline$>65$ & $23(21.9)$ & $1(11.1)$ & \\
\hline \multicolumn{4}{|l|}{ Sex } \\
\hline Male & $85(81.0)$ & $5(55.6)$ & 0.073 \\
\hline Female & $20(19.0)$ & $4(44.4)$ & \\
\hline \multicolumn{4}{|c|}{$\begin{array}{l}\text { Child-Pugh } \\
\text { classification }\end{array}$} \\
\hline A & $99(97.1)$ & $9(100)$ & 0.564 \\
\hline $\mathrm{B}$ & $3(2.9)$ & $0(0)$ & \\
\hline \multicolumn{4}{|c|}{ ICG-15 (\%) } \\
\hline$\leq 10$ & $71(68.3)$ & $4(44.4)$ & 0.147 \\
\hline$>10$ & $33(31.7)$ & $5(55.6)$ & \\
\hline \multicolumn{4}{|c|}{$\alpha$-fetoprotein $(\mathrm{ng} / \mathrm{ml})$} \\
\hline$\leq 200$ & $58(55.2)$ & $2(22.2)$ & 0.057 \\
\hline$>200$ & $47(44.8)$ & $7(77.8)$ & \\
\hline \multicolumn{4}{|l|}{ Size $(\mathrm{cm})$} \\
\hline$\leq 5$ & $85(81.0)$ & $6(66.7)$ & 0.305 \\
\hline$>5$ & $20(19.0)$ & $3(33.3)$ & \\
\hline \multicolumn{4}{|c|}{ LN metastasis } \\
\hline Yes & $0(0)$ & $2(22.2)$ & $<0.001$ \\
\hline No & $87(100)$ & $7(77.8)$ & \\
\hline \multicolumn{4}{|c|}{ CDH17 expression ${ }^{\mathrm{b}}$} \\
\hline Positive & $2(1.9)$ & $3(33.3)$ & $<0.001$ \\
\hline Negative & $102(98.1)$ & $6(66.7)$ & \\
\hline \multicolumn{4}{|c|}{ Encapsulation } \\
\hline Yes & $86(81.9)$ & $7(77.8)$ & 0.759 \\
\hline No & $19(18.1)$ & $2(22.2)$ & \\
\hline \multicolumn{4}{|c|}{ Vascular invasion } \\
\hline Yes & $25(23.8)$ & $4(44.4)$ & 0.173 \\
\hline No & $80(76.2)$ & $5(55.6)$ & \\
\hline \multicolumn{4}{|c|}{ Tumor rupture } \\
\hline Yes & $5(4.8)$ & $1(11.1)$ & 0.413 \\
\hline No & $100(95.2)$ & $8(88.9)$ & \\
\hline \multicolumn{4}{|c|}{ Daughter nodules } \\
\hline Yes & $19(18.1)$ & $3(33.3)$ & 0.266 \\
\hline No & $86(81.9)$ & $6(66.7)$ & \\
\hline \multicolumn{4}{|c|}{ Resection margin } \\
\hline Positive & $1(1.0)$ & $0(0)$ & 0.769 \\
\hline Negative & $104(99.0)$ & $9(100.0)$ & \\
\hline \multicolumn{4}{|c|}{$\begin{array}{l}\text { Edmonson and } \\
\text { steiner grade }\end{array}$} \\
\hline $\mathrm{I} / \mathrm{II}$ & $62(59.6)$ & $6(66.7)$ & 0.678 \\
\hline III/IV & $42(40.4)$ & $3(33.3)$ & \\
\hline \multicolumn{4}{|c|}{ Tumor necrosis } \\
\hline Yes & $52(49.5)$ & $4(44.4)$ & 0.770 \\
\hline No & $53(50.5)$ & $5(55.6)$ & \\
\hline
\end{tabular}

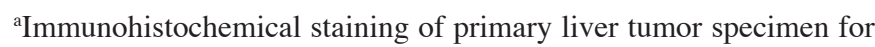
CK19. ' Immunohistochemical staining of primary liver tumor specimen for CDH17. ICG-15, indocyanine green retention at $15 \mathrm{~min}$; LN, lymph node; CDH17, cadherin 17; CK19, cytokeratin 19.
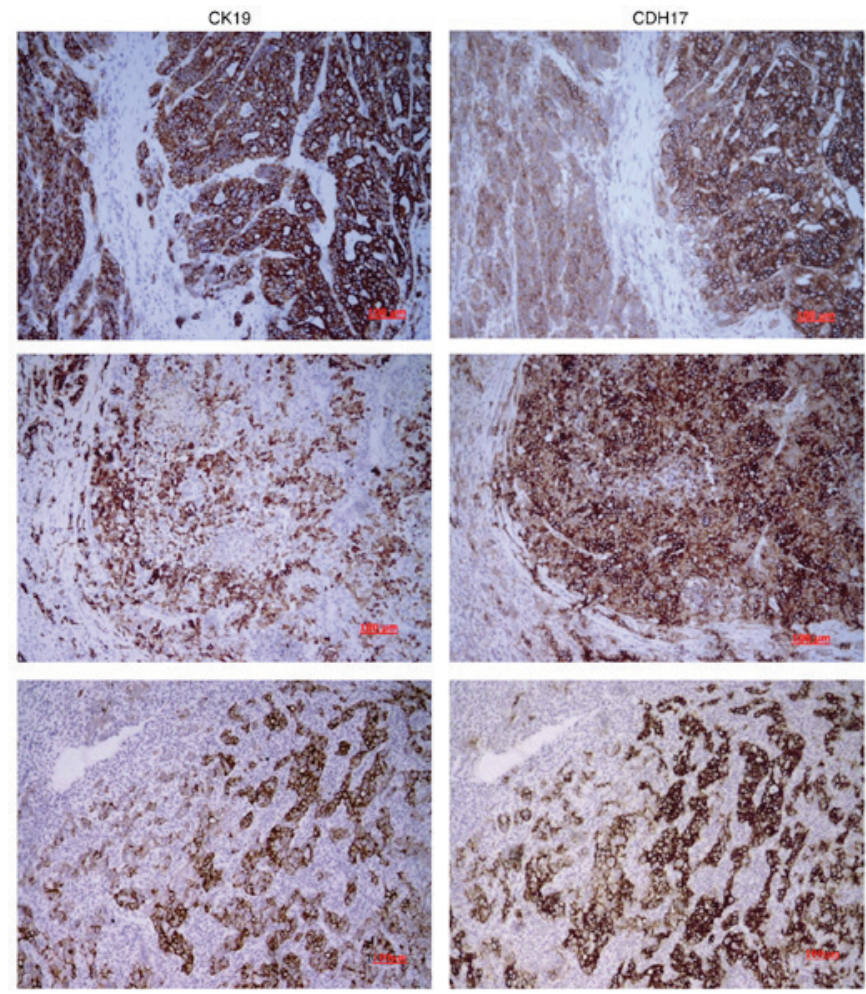

Figure 2. Immunohistochemical microphotograph of primary HBV-HCC. Two identical slides of tissue were sectioned from each tumor sample and then prepared for IHC study as described. The left column probed for CK19 and right column probed for CDH17. The expression of CK19 seemed to coincide with that of CDH17 in these HBV-HCC. The vast majority of the CK19(+) tumor cells in these HCC also had strong CDH17 expression. The Spearman's correlation coefficient between CK19 and CDH17 was 0.414, $\mathrm{P}<0.001$. Magnifications, $\mathrm{x} 100$. HBV, hepatitis B virus; $\mathrm{HCC}$, hepatocellular carcinoma; IHC, immunohistochemistry; CK19, cytokeratin 19; CDH17, cadherin 17.

( $\mathrm{P}<0.001$, respectively). Moreover, CDH17(+) HCC was more likely to be poorly-differentiated tumors and have vascular invasion (Table III).

As for survival analysis, CK19(+) HCC had a mean DFS of only $27.3 \pm 10.33$ months, while CK19(-) HCC could remain disease free for a mean of $54.7 \pm 3.75$ months $(\mathrm{P}=0.037)$. CDH17 expression was also a significantly poor prognostic factor for DFS in HCC, with CDH17(+) HCC having a mean DFS of only $8.8 \pm 4.86$ months (vs. $54.4 \pm 3.70$ months for CDH17(-) HCC, P<0.001). In CK19(+) HCC, CDH17 expression seemed to further promote tumor recurrence that DFS was only $3.6 \pm 1.06$ months (vs. $54.4 \pm 3.68$ months for dual negative or single positive $\mathrm{HCC}, \mathrm{P}<0.001)$. Moreover, the early recurrence rate was extremely high if HCC expressed both CK19 and CDH17. The three dual-immunopositive HCC patients all had tumor relapse within 2 years after the operation, compared to only $36 \%$ (40 out of 111 patients) in dual negative or single positive patients $(\mathrm{P}=0.024)$. CK19 expression was also a poor prognostic factor for OS, with CK19(+) HCC having a mean OS of 52.6 \pm 10.83 months (vs. 81.5 \pm 2.62 months for CK19(-) HCC, P=0.017). CDH17 expression, on the other hand, was not significantly related to a worse OS $(53.4 \pm 15.32$

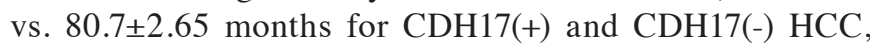
respectively, $\mathrm{P}=0.097)$. Fig. 3 demonstrated the DFS and OS of respective type of $\mathrm{HCC}$. 
Table III. Relationship between clinicopathological variables and CDH17 expression in hepatocellular carcinoma.

\begin{tabular}{|c|c|c|c|}
\hline \multicolumn{4}{|c|}{ Hepatocellular carcinoma $(n=114)$} \\
\hline \multirow[b]{2}{*}{ Variable } & \multicolumn{3}{|c|}{$\mathrm{CDH} 17^{\mathrm{a}}$} \\
\hline & Negative $(\%)$ & Positive $(\%)$ & P-value \\
\hline \multicolumn{4}{|c|}{ Age (years) } \\
\hline$\leq 65$ & $86(79.6)$ & $4(80.0)$ & 0.984 \\
\hline$>65$ & $22(20.4)$ & $1(20.0)$ & \\
\hline \multicolumn{4}{|l|}{ Sex } \\
\hline Male & $85(78.7)$ & $4(80.0)$ & 0.945 \\
\hline Female & $23(21.3)$ & $1(20.0)$ & \\
\hline \multicolumn{4}{|c|}{$\begin{array}{l}\text { Child-Pugh } \\
\text { classification }\end{array}$} \\
\hline A & $102(97.1)$ & $5(100)$ & 0.702 \\
\hline $\mathrm{B}$ & $3(2.9)$ & $0(0)$ & \\
\hline \multicolumn{4}{|l|}{ ICG-15 } \\
\hline$\leq 10$ & $72(67.3)$ & $2(40.0)$ & 0.208 \\
\hline$>10$ & $35(32.7)$ & $3(60.0)$ & \\
\hline \multicolumn{4}{|c|}{$\begin{array}{l}\alpha \text {-fetoprotein } \\
(\mathrm{ng} / \mathrm{ml})\end{array}$} \\
\hline$\leq 200$ & $58(53.7)$ & $2(40.0)$ & 0.548 \\
\hline$>200$ & $50(46.3)$ & $3(60.0)$ & \\
\hline \multicolumn{4}{|l|}{ Size $(\mathrm{cm})$} \\
\hline$\leq 5$ & $86(79.6)$ & $4(80.0)$ & 0.984 \\
\hline$>5$ & $22(20.4)$ & $1(20.0)$ & \\
\hline \multicolumn{4}{|c|}{ LN metastasis } \\
\hline Yes & $0(0)$ & $2(40.0)$ & $<0.001$ \\
\hline No & $89(100)$ & $3(60.0)$ & \\
\hline \multicolumn{4}{|c|}{ CK19 expression ${ }^{b}$} \\
\hline Positive & $6(5.6)$ & $3(60.0)$ & $<0.001$ \\
\hline Negative & $102(94.4)$ & $2(40.0)$ & \\
\hline \multicolumn{4}{|c|}{ Encapsulation } \\
\hline Yes & $90(83.3)$ & $3(60.0)$ & 0.296 \\
\hline No & $18(16.7)$ & $2(40.0)$ & \\
\hline \multicolumn{4}{|c|}{ Vascular invasion } \\
\hline Yes & $26(24.1)$ & $3(60.0)$ & 0.072 \\
\hline No & $82(75.9)$ & $2(40.0)$ & \\
\hline \multicolumn{4}{|c|}{ Tumor rupture } \\
\hline Yes & $5(4.6)$ & $1(20.0)$ & 0.134 \\
\hline No & $103(95.4)$ & $4(80.0)$ & \\
\hline \multicolumn{4}{|c|}{ Daughter nodules } \\
\hline Yes & $20(18.5)$ & $1(20.0)$ & 0.934 \\
\hline No & $88(81.5)$ & $4(80.0)$ & \\
\hline \multicolumn{4}{|c|}{ Resection margin } \\
\hline Positive & $1(0.9)$ & $0(0)$ & 0.829 \\
\hline Negative & $107(99.1)$ & $5(100)$ & \\
\hline \multicolumn{4}{|c|}{ Edmonson and } \\
\hline \multicolumn{4}{|c|}{ Steiner grade } \\
\hline $\mathrm{I} / \mathrm{II}$ & $67(62.6)$ & $1(20.0)$ & 0.057 \\
\hline III/IV & $40(37.4)$ & $4(80.0)$ & \\
\hline \multicolumn{4}{|c|}{ Tumor necrosis } \\
\hline Yes & $52(48.1)$ & $4(80.0)$ & 0.164 \\
\hline No & $56(51.9)$ & $1(20.0)$ & \\
\hline
\end{tabular}

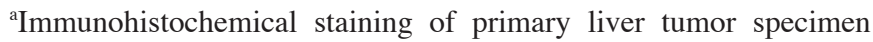
for CDH17. 'Immunohistochemical staining of primary liver tumor specimen for CK19. CDH17, cadherin 17; CK19, cytokeratin 19; ICG-15, indocyanine green retention at $15 \mathrm{~min}$; LN, lymph node.
Table IV. Cox regression multivariate analyses of factors associated with disease-free survival in hepatocellular carcinoma after hepatectomy.

\begin{tabular}{llc}
\hline & \multicolumn{2}{c}{ Multivariate analysis } \\
\cline { 2 - 3 } Variable & Hazard ratio (95\% CI) & P-value \\
\hline ICG-15, >10\% & $2.375(1.230-4.587)$ & 0.010 \\
Tumor size, $>5 \mathrm{~cm}$ & $1.405(0.515-3.840)$ & 0.506 \\
Vascular invasion & $1.474(0.434-5.008)$ & 0.534 \\
Daughter nodule & $1.664(0.640-4.326)$ & 0.296 \\
AJCC T stage & & 0.422 \\
T2 vs. T1 & $1.794(0.578-5.565)$ & 0.312 \\
T3a vs. T1 & $2.897(0.423-19.843)$ & 0.279 \\
T3b vs. T1 & $9.842(0.922-105.059)$ & 0.058 \\
T4 vs. T1 & $1.474(0.248-8.741)$ & 0.669 \\
AJCC N stage & $2.813(0.132-59.960)$ & 0.508 \\
CK19 expression & $2.859(0.917-8.908)$ & 0.070 \\
CDH17 expression & $8.894(2.280-34.701)$ & 0.002 \\
\hline
\end{tabular}

ICG-15, indocyanine green retention at $15 \mathrm{~min}$; AJCC, American Joint Committee on Cancer; CK19, cytokeratin 19; CDH17, cadherin 17; CI, confidence interval.

Table V. Cox regression multivariate analyses of factors associated with overall survival in hepatocellular carcinoma after hepatectomy.

\begin{tabular}{lcc}
\hline & \multicolumn{2}{c}{ Multivariate analysis } \\
\cline { 2 - 3 } Variable & Hazard ratio $(95 \%$ CI $)$ & P-value \\
\hline Male sex & $10.088(1.253-81.197)$ & 0.030 \\
ICG-15, >10\% & $2.530(0.938-6.820)$ & 0.067 \\
Tumor size, $>5 \mathrm{~cm}$ & $2.385(0.770-7.386)$ & 0.132 \\
Tumor rupture & $1.387(0.255-7.527)$ & 0.705 \\
Vascular invasion & $1.941(0.716-5.265)$ & 0.193 \\
Daughter nodule & $1.869(0.696-5.021)$ & 0.215 \\
Tumor necrosis & $3.246(0.933-11.288)$ & 0.064 \\
CK19 expression & $4.480(1.066-18.838)$ & 0.041 \\
\hline
\end{tabular}

ICG-15, indocyanine green retention at $15 \mathrm{~min}$; CK19, cytokeratin 19; $\mathrm{CI}$, confidence interval.

After Cox regression multivariate analysis, we further demonstrated that CDH17 expression and ICG-15 >10\% were the most significant independent poor prognostic factors for DFS ( $\mathrm{P}=0.010$ and 0.002 , respectively) (Table IV). Male sex and CK19 expression, on the other hand, were independent poor prognostic factors for $\mathrm{OS}(\mathrm{P}=0.030$ and 0.041 , respectively) (Table V).

Causal relationship between $\mathrm{CK} 19$ and $\mathrm{CDH} 17$ in HCC. To further explore the causal relationship between CK19 and CDH17 expressions in HCC, various HCC cell lines were 

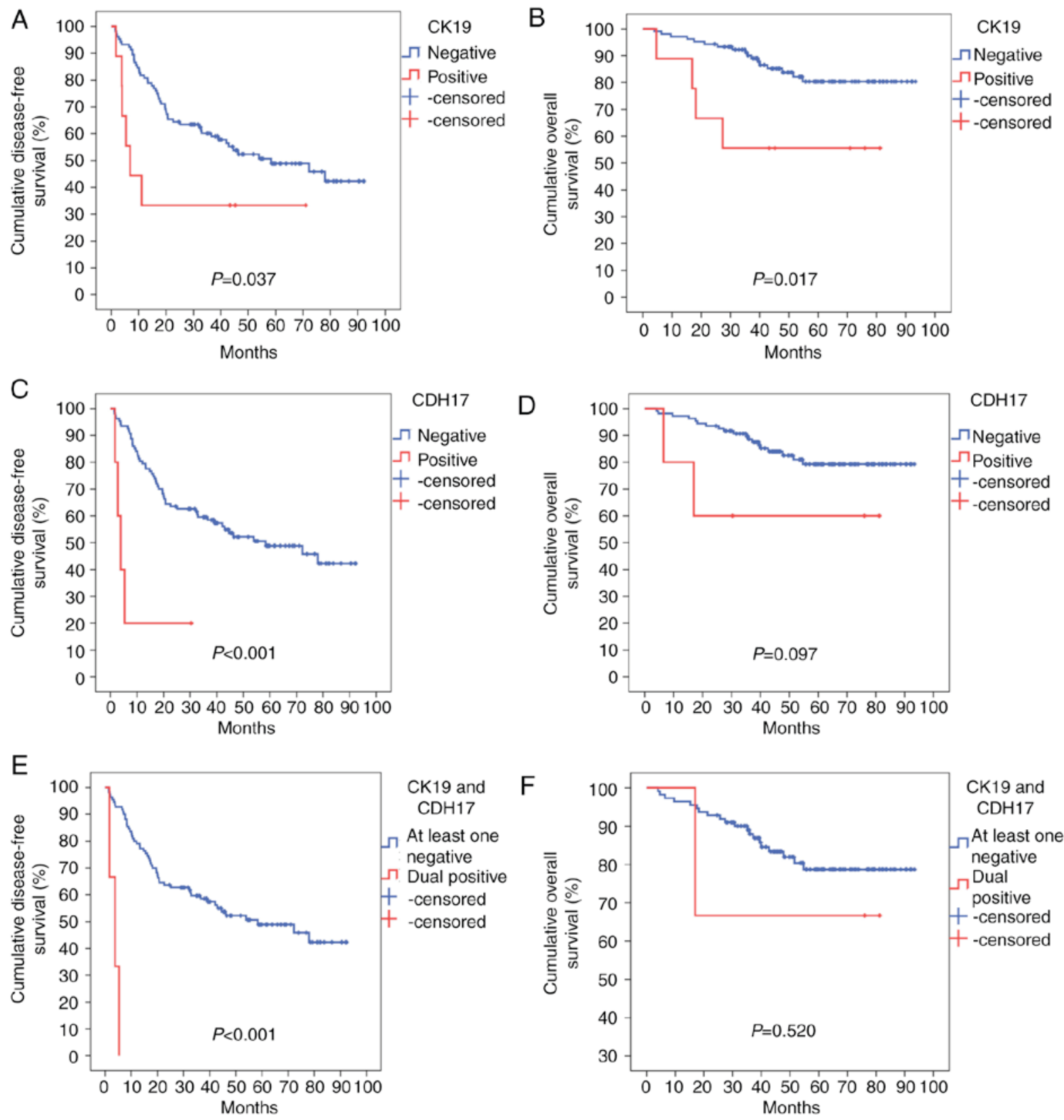

Figure 3. Kaplan-Meier survival curves. (A and B) HCC with CK19 expression had a significantly poorer prognosis in terms of (A) DFS and (B) OS; (C) HCC with CDH17 expression had a significantly worse DFS. (D) However, the OS was comparable to those without CDH17 expression; (E) HCC with dual CK19 and CDH17 expressions had a worst prognosis in terms of DFS. (F) The OS was comparable to those with either single positive or dual negative HCC. HCC, hepatocellular carcinoma; CK19, cytokeratin 19; DFS, disease-free survival; OS, overall survival; CDH17, cadherin 17.

employed to conduct in vitro studies. The expression profiles of CK19 and CDH17 in HCC cell lines including Huh7, Hep3B, HepG2, and PLC5 were determined by Western blot analysis. As shown in Fig. 4A, Hep3B, Huh7, and PLC5 were strongly positive for CK19 expression, while HepG2 was essentially a CK19(-) HCC cell line. On the other hand, Hep3B and Huh7 had strong CDH17 expression, while HepG2 and PLC5 only had faint CDH17 expression. To induce CK19 expression in CK19(-) HCC cell line, epidermal growth factor (EGF) was treated to HepG2 cell line and real-time qPCR as well as western blot analysis were performed (7). As shown in Fig. 4B and $\mathrm{C}$, the expression of CK19 was enhanced significantly after EGF treatment, and that of $\mathrm{CDH} 17$ was also increased dramatically under EGF treatment $(\mathrm{P}<0.001$, respectively). To further dissect the relationship between these two molecules, CK19 or CDH17 was overexpressed in HepG2 and the result was demonstrated in Fig. 5. The transfection of FLAG ${ }^{\circledR}-\mathrm{CDH} 17$ into HepG2 resulted in enhanced expression of both $\mathrm{CDH} 17$ and CK19. The mRNA transcripts of CDH17 and CK19 increased by 598,918 folds and 2.32 folds, respectively, after transfection ( $\mathrm{P}=0.03$ and $<0.001$, respectively) (Fig. 5A and $\mathrm{B}$ ). On the other hand, while both CK19 mRNA and protein levels were enhanced significantly after $F L A G^{\circledR}-\mathrm{CK} 19$ transfection in HepG2 $(\mathrm{P}<0.001)$, the level of $\mathrm{CDH} 17$ remained unchanged after the transfection (Fig. 5C and D). The result indicated that $\mathrm{CDH} 17$ was not downstream to CK19. In contrast, it was upstream to CK19 and, like CK19, was regulated by a common signal such as EGF. 

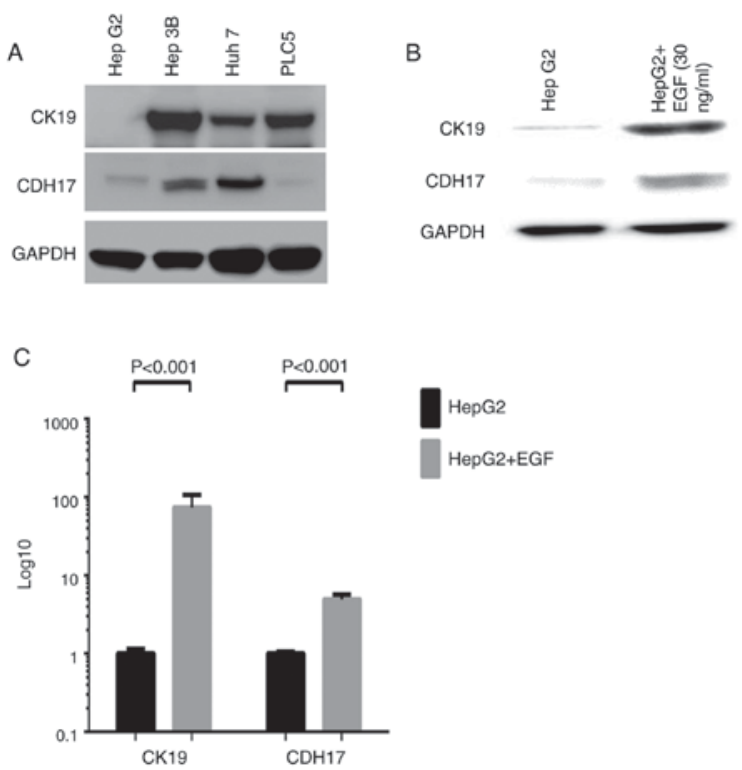

Figure 4. (A) Western blot analysis of various HCC cell lines. Primary antibodies were that of CK19 and CDH17 and a dilution of 1:100,000 and 1:2,000, respectively, were adopted. GAPDH was used as internal control. Hep3B, Huh7, and PLC5 were CK19(+) HCC cell lines, while HepG2 was CK19(-) HCC cell line. On the other hand, Hep3B and Huh7 were CDH17(+) HCC cell lines, while HepG2 and PLC5 were CDH17(-) HCC cell lines. (B and C) The study of EGF-treated HepG2 cells (EGF, $30 \mathrm{ng} / \mathrm{ml}$ for 5 days). Western blot analysis confirmed that CK19 can be induced by EGF treatment. In the meanwhile, the expression of CDH17 was also enhanced after EGF treatment (B). Real-time qPCR analysis of EGF-treated HepG2 cells demonstrated that both CK19 and CDH17 transcripts were significantly elevated (P<0.001 and $<0.001$, respectively) $(\mathrm{C})$. In the other words, EGF can induce both CK19 and CDH17 expressions. HCC, hepatocellular carcinoma; CK19, cytokeratin 19; CDH17, cadherin 17; GAPDH, glyceraldehyde-3-phosphate dehydrogenase; EGF, epidermal growth factor.

A

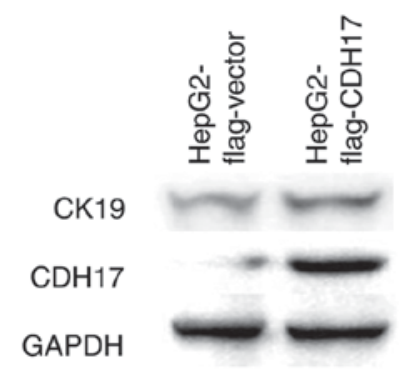

C

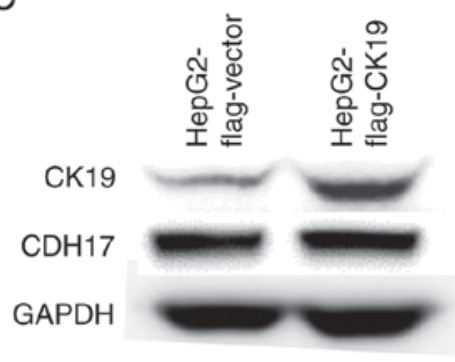

B

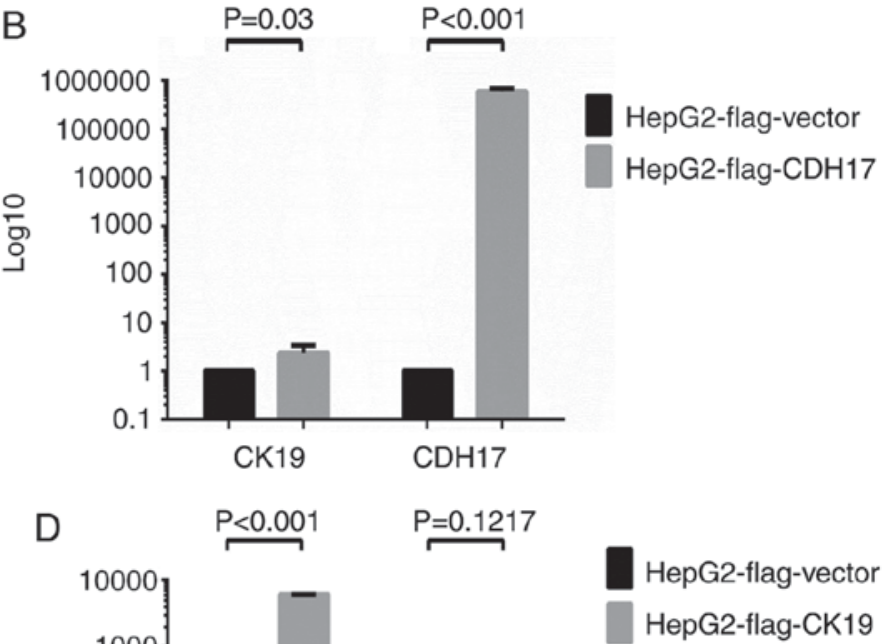

Figure 5. Over-expression assays to study causal relationships. (A and B) Over-expression study by transfecting HepG2 cells with FLAG ${ }^{\circledR}$-CDH17 [pCMV6-Entry $\left(\right.$ OriGene ${ }^{\mathrm{TM}}$ ) with CDH17 gene incorporated] or FLAG ${ }^{\circledR}$-vector [pCMV6-Entry (OriGene ${ }^{\mathrm{TM}}$ )]. Western blot analysis showed that the expression of both CDH17 and CK19 increased significantly after transfecting HepG2 with FLAG ${ }^{\circledR}$-CDH17 (A). Real-time qPCR revealed that the transcripts of both CDH17 and CK19 were significantly increased in HepG2 $\mathrm{FLAG}^{\circledR}-\mathrm{CDH} 17$ cells $(\mathrm{P}<0.001$ and 0.03 , respectively) (B). (C and D) Over-expression study by transfecting HepG2 cells with FLAG ${ }^{\circledR}-\mathrm{CK} 19$ [pCMV-3Tag-8 (Invitrogen ${ }^{\mathrm{TM}}$ ) with CK19 gene incorporated] or FLAG ${ }^{\circledR}$-vector [pCMV-3Tag-8 (Invitrogen ${ }^{\mathrm{TM}}$ )]. Western blot analysis showed that the expression of CK19 increased significantly after transfecting with FLAG ${ }^{\circledR}$-CK19. However, the expression of CDH17 remained unchanged after CK19 over-expression (C). Real-time qPCR revealed that the transcripts of CK19 were significantly increased in HepG2 LLAG ${ }^{\circledR}$-CK19 cells $(\mathrm{P}<0.001)$. However, the transcripts of CDH17 were not altered by CK19 over-expression (P=0.1217) (D). In other words, CDH17 was not downstream to CK19. It was upstream to CK19 and, like CK19, was regulated by EGF. The experiment of real-time qPCR was conducted in triplicates. CDH17, cadherin 17; CK19, cytokeratin 19; EGF, epidermal growth factor. 


\section{Discussion}

CK19 is generally believed to be a marker for biliary epithelial cells. Tumor cells from HCC, as a result, should not express CK19 since they are supposed to originate from hepatocytes (16). However, previous studies also showed that about $10 \%$ of HCC with typical microscopic histologic features expressed CK19 (4,6). These CK19(+) HCC was found to behave more aggressively and had a worse outcome $(4,6-8,17)$. For example, Takano et al demonstrated that CK19(+) HCC exhibited higher invasiveness, metastatic potential, and poorer prognosis (17). Our previous study also illustrated that CK19 expression in primary liver tumor was associated with LNM, tumor non-encapsulation, and worse OS (4). The current study verified that CK19 expression in HCC was associated with LNM, poorer DFS, and worse OS. Due to distinct clinical behavior and poor outcome, CK19(+) HCC may be a special subtype of HCC that deserves further investigation. Unfortunately, few studies to date had tried to dissect the possible mechanism responsible for poor prognosis in CK19(+) HCC. A previous research indicated that the activation of the EGF-epidermal growth factor receptor (EGFR) signaling pathway was associated with the development of CK19-positive HCC, and the EGF-induced increase in growth abilities of HCC might account for the poor prognosis of the patients (7). A recent study identified that the poor clinical outcome of CK19(+) HCC may be attributed to the effect of CK19 on angiogenesis-related molecules such as vasohibin-1 (VASH1) and fibroblast growth factor 1 (FGFR1) (17). All of these studies attempt to unravel the molecular mechanism underlying CK19(+) HCC, and once the mechanism is identified and explored, a targeted therapy can be developed to deal with this subset of HCC.

In the current study, we identified that the expression of CDH17 was significantly associated with that of CK19 at both transcription and translation levels in HBV-HCC. Previous study has shown that $\mathrm{CDH} 17$ could be a biomarker for gastric carcinoma and attractive therapeutic target for this aggressive malignancy (18). Recent reports also demonstrated that the expression of CDH17 in HCC was associated with vascular invasion, tumor metastasis, advanced tumor stage, and poor prognosis $(12,13,19,20)$. The therapeutic significance was determined as well that, by targeting CDH17, we can inhibit tumor growth in HCC $(13,19)$. Despite numerous works and reports, however, no study to date has dealt with the involvement of CDH17 in CK19(+) HCC. Our study is by far the first one to explore the relationship between CK19 and CDH17 in HBV-HCC.

The present study demonstrated that $\mathrm{CDH} 17$ expression was one of the most significant poor prognostic factors for DFS in HBV-HCC. The CDH17(+) HCC patients is expected to have tumor relapse within 9 months after the operation. For patients who were treated with curative hepatectomy, early recurrence was proved to be a poor prognostic factor for OS (21). In addition, CDH17 expression was associated with LNM and a likelihood of vascular invasion in HCC. Both LNM and vascular invasion were well known poor prognostic factors for DFS and OS after hepatectomy (11). Our study, as a result, agreed with previous reports that $\mathrm{CDH} 17$ expression was a poor prognostic factor for HCC after hepatectomy. Given substantial correlation between CK19 and CDH17 expressions, we believe that the high recurrence rate and poor prognosis of CK19(+) HCC may be attributed to CDH17 expression. Since CDH17 has been reported to influence HCC outcome by targeting Wnt/ß-catenin pathway, anti-CDH17 Ab or inhibitors of Wnt $/ \beta$-catenin pathway may have promising effects for CK19(+) HCC (18-20).

In an attempt to dissect the causal relationship between CK19 and CDH17, we conducted in vitro studies and confirmed that CK19 can be induced by EGF treatment. In addition to CK19, CDH17 was also induced upon EGF stimulation. Therefore, we believe that in CK19(+) HCC, EGF/EGFR may act as an initiator that induce the expression of both CK19 and $\mathrm{CDH} 17$, resulting in worst prognosis. EGF/EGFR is responsible for a variety of cellular functions and pathologic processes including cell cycle progression, gene transcription, cell proliferation, migration, and adhesion $(22,23)$. The exact mechanism by which EGF/EGFR influences CK19 and CDH17 expressions mandates further investigations. Last but not the least, by establishing a stable CDH17 transfectant in HepG2, we found in the current study that the expression of CK19 was enhanced by $\mathrm{CDH} 17$ over-expression. CK19, as a result, was regulated by $\mathrm{CDH} 17$ in HCC. Our novel findings explains the significant association between CDH17 and CK19 in HBV-HCC, and we believe that the poor prognosis of CK19(+) HCC is partly attributed to this causal relationship. The current study, subsequently, should be the first one in the English literature to identify this EGF/EGFR-CDH17-CK19 pathway in HCC. Treatment targeted against EGF/EGFR or $\mathrm{CDH} 17$ may thus be beneficial for CK19(+) HCC. Further studies are still warranted to validate our findings and to explore the detailed mechanistic relationships between these molecules.

In conclusion, our study demonstrated that CK19(+) HCC was significantly associated with LNM and a worse survival. The expression of CDH17 was significantly associated with that of CK19 in HBV-HCC. CDH17, a gene known to be associated with vascular invasion, tumor metastasis, and advanced tumor stage, was found to have an extremely poor DFS in the current study. As a result, we believe that in CK19(+) HCC, $\mathrm{CDH} 17$ plays an important role in promoting tumor recurrence and leading to poor prognosis. Lastly, we found that EGF can induce the expressions of both $\mathrm{CK} 19$ and $\mathrm{CDH} 17$, and CDH17 in turn can enhance the expression of CK19 in HCC. Novel therapeutics by targeting EGF/EGFR or CDH17 may thus be beneficial for CK19(+) HCC. Further study is warranted to determine the detailed mechanistic relationships between these molecules.

\section{Acknowledgements}

We are grateful to all our colleagues in the Department of Cancer Center, Department of Pathology, the Genomic Medicine Research Core Laboratory of Chang Gung Memorial Hospital, and Graduate Institute of Clinical Medical Sciences, Chang Gung University for their technical assistance. We are also grateful to Chun-Hsing Wu, Shu-Chuan Yu, and Jo-Chu Chiu for their laboratory assistance. This study was supported by Chang Gung Memorial Hospital (CMRPG3G0051) and Ministry of Science and Technology, Taiwan, R.O.C. (NMRPG3F0321). 


\section{References}

1. Torre LA, Bray F, Siegel RL, Ferlay J, Lortet-Tieulent J and Jemal A: Global cancer statistics, 2012. CA Cancer J Clin 65: 87-108, 2015.

2. Sartorius K, Sartorius B, Aldous C, Govender PS and Madiba TE: Global and country underestimation of hepatocellular carcinoma (HCC) in 2012 and its implications. Cancer Epidemiol 39: 284-290, 2015.

3. Ministry of Health and Welfare, Executive Yuan, R.O.C: Report of leading cancer-related death in 2014. 2015.

4. Lee CW, Kuo WL, Yu MC, Chen TC, Tsai CN, Lee WC and Chen MF: The expression of cytokeratin 19 in lymph nodes was a poor prognostic factor for hepatocellular carcinoma after hepatic resection. World J Surg Oncol 11: 136, 2013.

5. Corcelle V, Stieger B, Gjinovci A, Wollheim CB and Gauthier BR: Characterization of two distinct liver progenitor cell subpopulations of hematopoietic and hepatic origins. Exp Cell Res 312 2826-2836, 2006

6. Yamamoto T, Uenishi T, Ogawa M, Ichikawa T, Hai S, Sakabe K, Tanaka S, Kato H, Mikami S, Ikebe T, et al: Immunohistologic attempt to find carcinogenesis from hepatic progenitor cell in hepatocellular carcinoma. Dig Surg 22: 364-370, 2005.

7. Yoneda N, Sato Y, Kitao A, Ikeda H, Sawada-Kitamura S, Miyakoshi M, Harada K, Sasaki M, Matsui O and Nakanuma Y: Epidermal growth factor induces cytokeratin 19 expression accompanied by increased growth abilities in human hepatocellular carcinoma. Lab Invest 91: 262-272, 2011.

8. Hoshida Y, Toffanin S, Lachenmayer A, Villanueva A, Minguez B and Llovet JM: Molecular classification and novel targets in hepatocellular carcinoma: Recent advancements. Semin Liver Dis 30: 35-51, 2010

9. Uenishi T, Hirohashi K, Shuto T, Kubo S, Tanaka H, Sakata C, Ikebe $\mathrm{T}$ and Kinoshita $\mathrm{H}$ : The clinical significance of lymph node metastases in patients undergoing surgery for hepatocellular carcinoma. Surg Today 30: 892-895, 2000

10. Chu KM, Lai EC, Al-Hadeedi S, Arcilla CE Jr, Lo CM, Liu CL, Fan ST and Wong J: Intrahepatic cholangiocarcinoma. World J Surg 21: 301-305; discussion 305-306, 1997.

11. Lee CW, Chan KM, Lee CF, Yu MC, Lee WC, Wu TJ and Chen MF: Hepatic resection for hepatocellular carcinoma with lymph node metastasis: Clinicopathological analysis and survival outcome. Asian J Surg 34: 53-62, 2011.

12. Ding ZB, Shi YH, Zhou J, Shi GM, Ke AW, Qiu SJ, Wang XY, Dai Z, Xu Y and Fan J: Liver-intestine cadherin predicts microvascular invasion and poor prognosis of hepatitis B virus-positive hepatocellular carcinoma. Cancer 115: 4753-4765, 2009.
13. Lee NP, Poon RT, Shek FH, Ng IO and Luk JM: Role of cadherin-17 in oncogenesis and potential therapeutic implications in hepatocellular carcinoma. Biochim Biophys Acta 1806: $138-145,2010$.

14. International Union Against Cancer (UICC): TNM Classification of Malignant Tumours. Sobin LH, Gospodarowicz MK and Wittekind C (eds). 7th edition. Wiley-Blackwell, Hoboken, NJ, 2009.

15. Yu MC, Lee YS, Lin SE, Wu HY, Chen TC, Lee WC, Chen MF and Tsai CN: Recurrence and poor prognosis following resection of small hepatitis B-related hepatocellular carcinoma lesions are associated with aberrant tumor expression profiles of glypican 3 and osteopontin. Ann Surg Oncol 3 (Suppl 19): S455-S463, 2012.

16. Libbrecht L, Desmet V, Van Damme B and Roskams T: The immunohistochemical phenotype of dysplastic foci in human liver: Correlation with putative progenitor cells. J Hepatol 33: 76-84, 2000 .

17. Takano M, Shimada K, Fujii T, Morita K, Takeda M, Nakajima Y, Nonomura A, Konishi N and Obayashi C: Keratin 19 as a key molecule in progression of human hepatocellular carcinomas through invasion and angiogenesis. BMC Cancer 16: 903, 2016.

18. Qiu HB, Zhang LY, Ren C, Zeng ZL, Wu WJ, Luo HY, Zhou ZW and $\mathrm{Xu} \mathrm{RH}$ : Targeting $\mathrm{CDH} 17$ suppresses tumor progression in gastric cancer by downregulating Wnt/ $\beta$-catenin signaling. PLoS One 8: e56959, 2013.

19. Liu LX, Lee NP, Chan VW, Xue W, Zender L, Zhang C, Mao M, Dai H, Wang XL, Xu MZ, et al: Targeting cadherin-17 inactivates Wnt signaling and inhibits tumor growth in liver carcinoma. Hepatology 50: 1453-1463, 2009.

20. Wang Y, Shek FH, Wong KF, Liu LX, Zhang XQ, Yuan Y, Khin E, Hu MY, Wang JH, Poon RT, et al: Anti-cadherin-17 antibody modulates beta-catenin signaling and tumorigenicity of hepatocellular carcinoma. PLoS One 8: e72386, 2013.

21. Yamamoto Y, Ikoma H, Morimura R, Konishi H, Murayama Y, Komatsu S, Shiozaki A, Kuriu Y, Kubota T, Nakanishi M, et al: Optimal duration of the early and late recurrence of hepatocellular carcinoma after hepatectomy. World J Gastroenterol 21: 1207-1215, 2015

22. Seshacharyulu P, Ponnusamy MP, Haridas D, Jain M, Ganti AK and Batra SK: Targeting the EGFR signaling pathway in cancer therapy. Expert Opin Ther Targets 16: 15-31, 2012.

23. Blobel CP: ADAMs: Key components in EGFR signalling and development. Nat Rev Mol Cell Biol 6: 32-43, 2005. 